\title{
Polyphenolic Herbal Extract of Cistus incanus as Natural Preservatives for Sausages Enriched with Natural Colors
}

\author{
Marta Sośnicka ${ }^{1, *}$, Agnieszka Nowak ${ }^{1} \mathbb{1}$, Agata Czyżowska $^{1}$ (D) , Ilona Gałązka-Czarnecka ${ }^{2}$ \\ and Aleksandra Czerbniak ${ }^{1}$ \\ 1 Institute of Fermentation Technology and Microbiology, Lodz University of Technology, Wolczanska 171/173, \\ 90-530 Lodz, Poland; agnieszka.nowak@p.lodz.pl (A.N.); agata.czyzowska@p.lodz.pl (A.C.); \\ 212004@edu.p.lodz.pl (A.C.) \\ 2 Institute of Food Technology and Analysis, Lodz University of Technology, Stefanowskiego 4/10, \\ 90-924 Lodz, Poland; ilona.galazka-czarnecka@p.lodz.pl \\ * Correspondence: marta.sosnicka@dokt.p.lodz.pl
}

check for updates

Citation: Sośnicka, M.; Nowak, A.; Czyżowska, A.; Gałązka-Czarnecka, I.; Czerbniak, A. Polyphenolic Herbal Extract of Cistus incanus as Natural Preservatives for Sausages Enriched with Natural Colors. Processes 2021, 9 , 1556. https://doi.org/10.3390/pr 9091556

Academic Editor: Joanna

Kobus-Cisowska

Received: 17 August 2021

Accepted: 27 August 2021

Published: 31 August 2021

Publisher's Note: MDPI stays neutral with regard to jurisdictional claims in published maps and institutional affiliations.

Copyright: (c) 2021 by the authors. Licensee MDPI, Basel, Switzerland. This article is an open access article distributed under the terms and conditions of the Creative Commons Attribution (CC BY) license (https:// creativecommons.org/licenses/by/ $4.0 /)$.

\begin{abstract}
This study evaluates the effects of polyphenolic extract of Cistus incanus, lycopene dye from tomatoes, and betanin dye from red beet on selected parameters of model meat products with reduced nitrate contents. The polyphenolic composition and activity of the $C$. incanus extract was analyzed, revealing the presence of elagotannins, flavanols, and glycosylated flavanols. We studied the effects of the extract and dyes as well as of mixtures of the extract and dyes on the growth of bacteria characteristic of the meat environment: E. coli, S. enterica, P. fragi, L. monocytogenes, B. thermosphacta, and L. sakei. We studied the effects of the extract and dyes on the lipid oxidation, color, and microbiological quality of pork sausages with reduced nitrate content over 28 days of storage. During storage, the amounts of malon dialdehyde reduced, which indicates that the extract and dyes exhibited antioxidant activity and slowed lipid oxidation in the sausages. An increase in red color was also observed in the sausages with natural additives, despite their decreased nitrate content. It was found that the $C$. incanus extract combined with coloring agents positively influenced the selected parameters of the analyzed pork sausages.
\end{abstract}

Keywords: Cistus incanus; lycopene; beetroot; polyphenols; natural preservatives; meat

\section{Introduction}

Curing processes used for the production of sausages in the meat industry involve the addition of salt, nitrites, nitrate salts, and spices to preserve and enhance the flavor, texture, and color of the final products [1]. Nitrates are artificial compounds added to processed meat products to inhibit the growth of pathogenic bacteria such as Listeria monocytogenes and Clostridium botulinum. Nitrates also prevent spoilage by retarding lipid oxidation and impart characteristic flavor and pink-red coloration to cured meats [2]. Despite their many benefits, nitrates may form carcinogenic N- nitroso compounds (NOCs), in particular nitrosamines, which increase the risk of esophageal, gastric, and bladder cancers [3]. In recent years, there has been growing consumer concern over the health risks of nitrates [1,4]. There has also been increasing consumer interest in healthy meat products enriched with natural ingredients $[5,6]$. Possible alternatives to nitrates include plant extracts and essential oils that contain bioactive compounds -polyphenols, flavonoids, and terpenoids characterized by strong antioxidant and antimicrobial activity $[4,7,8]$. Research using plant and vegetable extracts has been ongoing for several years $[9,10]$. It has been shown that natural extracts can be used as additives in the meat industry [4,7].

Color is one of the most important quality characteristics in the food industry. It signals that the correct technological processes have been implemented [11]. Meat coloration is also a sensory indicator that provides consumers with information about quality and freshness [12]. If the color is not acceptable by the consumer, then other characteristics such 
as consistency or taste lose interest [13]. Color is also an indicator of various properties of products, including nutritional value and food safety [14]. There has been considerable research conducted to optimize the addition of nitrates to cured products and replace them with other natural compounds [11].

Many natural additives can be used by the meat industry that have health-promoting properties [15]. Tabaka and co-authors prepared model meat products using sweet red peppers, cayenne pepper, rosehips, acai berries, cranberries and grape seeds as additives, to reduce the content of sodium nitrate III. The use of dyes imparted an attractive color despite the reduction in nitrate III. Products containing acai berry and grape seed pigments had the most stable color [16]. Dias and co-authors obtained cooked ham with a pink color using natural extracts from red radish, hibiscus, and red beetroot. Both the red beet extract and the hibiscus extract provided the desired color, but a higher quantity of the hibiscus dye was required [17]. Other researchers have tested the effect of adding lycopene from tomatoes to ground meat. The ground meat showed increased stability during storage. Good color characteristics were obtained, and the additive enhanced the meat product with documented health-promoting properties [18].

The aim of this study was to determine the effects of aqueous extract of Cistus incanus separately and in combination with natural lycopene dye from tomatoes and betanin dye from red beet. Cistus incanus is a Mediterranean plant with pink or white flowers depending on the species, which is found in North African and Southern European countries and is able to grow in difficult climates and soil conditions. Folk medicine has used C. incanus in the form of infusions to treat many diseases. Cistus incanus extracts are characterized by antimicrobial, antiviral, antifungal, and antibacterial activities [19-21]. Aqueous extracts of $C$. incanus actively inhibit the growth of human epithelial prostate cells [22]. We investigated the effects of the extract and dyes on the oxidative stability of fat, color, as well as organoleptic and microbiological characteristics of sausages with limited amounts of nitrate III. According to the best of our knowledge, no studies have been conducted on the effect of $C$. incanus extract on nitrate-reduced meat products. The effect of Cistus incanus extract, dyes, extract and dyes together on bacterial growth parameters has not been researched yet.

\section{Materials and Methods}

The research materials consisted of aqueous plant extract of $C$. incanus prepared using commercially available dried plants from (Batom, Poland), powdered betanin dye from beetroot (Trzy ziarna, Poland), and lycopene dye from tomatoes (Chinese Herbs Health wish, China).

\subsection{Preparation of C. incanus Plant Extract}

Dried C. incanus $(2.0 \mathrm{~g})$ was weighed and extracted with $50 \mathrm{~mL}$ of distilled water for $1 \mathrm{~h}$ at room temperature. The sample was then centrifuged for $15 \mathrm{~min}$ at $4{ }^{\circ} \mathrm{C}$ at $7000 \mathrm{rpm}$. The supernatant was decanted and the microwave sterilized.

\subsection{Preparation of Dyes}

Lyophilized dye $(1.0 \mathrm{~g})$ was weighed and suspended using $100 \mathrm{~mL}$ of distilled water with a few drops of Tween 20 for $1 \mathrm{~h}$ at room temperature. The dye was subjected to microwave sterilization.

\subsection{Determination of Total Phenolics in C. incanus Using Folin-Ciocalteu Reagent}

The total polyphenol content in the $C$. incanus plant extract was determined by the Folin-Ciocalteu method. First, to $1 \mathrm{~mL}$ of the $C$. incanus extract was added $0.2 \mathrm{~mL}$ of Folin-Ciocalteu reagent, Next, $1 \mathrm{~mL}$ of $20 \%$ calcium carbonate and $2 \mathrm{~mL}$ of distilled water were added. The samples were incubated for $60 \mathrm{~min}$ in a dark place at room temperature. The absorbance was further measured at $\lambda=756 \mathrm{~nm}$ against the reagent sample. The 
polyphenol content was determined as the amount of gallic acid equivalents (mg GAE/L of extract).

\subsection{Antioxidant Activity (DPPH and ABTS)}

The antioxidant activity of the extract and dyes was determined using the DPPH radical method [23] and ABTS [23]. In the DPPH method, $1.95 \mathrm{~mL}$ of the reagent solution (2,2-diphenyl-1-picrylhydrazyl) and $50 \mu \mathrm{L}$ extract solution were used. The sample was incubated for $30 \mathrm{~min}$ at room temperature, after which time the absorbance was measured at $\lambda=515 \mathrm{~nm}$ against the reagent sample. Antioxidant activity according to the DPPH method was defined as the amount of trolox equivalents ( $\mathrm{mg}$ trolox $/ 100 \mathrm{~mL}$ ). Antioxidant activity was also determined according to the ABTS using ABTS reagent $\left(2,2^{\prime}\right.$-azino-bis(3ethylbenzothiazoline- 6 sulfonic acid)) by adding $30 \mu \mathrm{L}$ of extract solution to $3 \mathrm{~mL}$ ABTS solution [23]. The solution was then incubated for $15 \mathrm{~min}$ in a dark place at room temperature and the absorbance of the samples was measured at $\lambda=734 \mathrm{~nm}$ in distilled water. Antioxidant activity according to the ABTS method was defined as the amount of trolox equivalents ( $\mathrm{mg}$ trolox $/ 100 \mathrm{~mL}$ ).

\subsection{LC-MS Analysis of Polyphenols}

The samples were filtered through a $0.45 \mu \mathrm{m}$ membrane into vials. They were analyzed using an HPLC coupled to an LTQ Velos MS mass spectrometer. Chromatographic separation was conducted using a column operating at $45^{\circ} \mathrm{C}$. The mobile phase consisted of solvent A ( $1 \mathrm{~mL}$ formic acid and $1 \mathrm{~mL}$ deionized water) and solvent B ( $95 \%$ acetonitrile). A Hypersil Gold $150 \times 2.1$ column was used, particle size $1.9 \mu \mathrm{m}$. Elution conditions: $96 \%$ to $85 \%$ A for $8 \mathrm{~min}, 85 \%$ to $82 \%$ A for $12 \mathrm{~min}, 82 \%$ to $60 \%$ A for $40 \mathrm{~min}, 60 \%$ to $50 \%$ A for $4 \mathrm{~min}, 60 \%$ to $50 \%$ A for $3 \mathrm{~min}, 50 \%$ to $96 \%$ A for $2 \mathrm{~min}$, further washing and rebalancing of the column. The mass spectra were recorded over $60 \mathrm{~min}$. The flow rate was $220 \mu \mathrm{L} / \mathrm{min}$, and the injection volume was $10 \mu \mathrm{L}$. Electrospray ionization mass spectrometry was conducted using an LTQ Velos mass spectrometer equipped with an ESI interface and controlled by Excalibur software. The spray voltage was $4 \mathrm{kV}$, the shielding gas flow rate was 25 . The auxiliary gas flow rate was 10 , the desolvation temperature was $28^{\circ} \mathrm{C}$, and the source temperature was $35^{\circ} \mathrm{C}$. Mass spectra were acquired in the negative mode over the range $m / z 120-1000$. The I spray voltage was $4 \mathrm{kV}$. The sheath gas flow rate was 25 and the aux gas flow rate 10 . The desolvation temperature was $280^{\circ} \mathrm{C}$, and the source temperature $350^{\circ} \mathrm{C}$. Peak identification was performed by comparing the retention time and mass spectra to standards and spectra from the literature.

\subsection{Evaluation of the Activity of Extracts and Dyes against Selected Bacterial Strains}

The three Gram positive bacteria Listeria monocytogenes (ATCC 13992), Brochotrix thermosphacta (MMAP4 HQ8909431*), and Latilactobacillus sakei (ATCC 15521), as well as the three Gram negative strains Escherichia coli (ATCC 10536), Pseudomonas fragi (ATCC 4973), and Salmonella enterica (MCH1 MG911721*), were used in the study. The strains S. enterica and B. thermosphacta were from natural environments. The 16s RNA sequence numbers in GenBank are given in parentheses above. The selected bacterial strains were activated and incubated to obtain $10^{7} \mathrm{cfu} / \mathrm{mL}$. The samples were then diluted in saline solution to obtain concentrations of $10^{4} \mathrm{cfu} / \mathrm{mL}$. A TSB culture medium was prepared in conical flasks, to which the extract $(3,6$, and $12 \mathrm{~mL})$, dyes $(10 \mathrm{~mL})$, or mixtures of the extract ( 3 or 6 of or $12 \mathrm{~mL}$ ) and dyes $(10 \mathrm{~mL}$ ) were added, with inoculum to a concentration of $10^{4} \mathrm{cfu} / \mathrm{mL}$. The reference was without extract or dyes. Subsequently, deep seeding was performed for $0,4,6,24,30,48 \mathrm{~h}$ or, for certain strains, $72 \mathrm{~h}$. The cultures were incubated at $30^{\circ} \mathrm{C}$. The samples were diluted for seeding, and then the plates were flooded with PCA culture medium and incubated for $24 \mathrm{~h}$ at $30^{\circ} \mathrm{C}$. The bacterial colonies were counted. The Excel add-on DmFit 3.5 was used and the growth parameters were calculated on the basis of the number of bacterial cells, by substituting under the Gompertz equation 
$\mathrm{L}(\mathrm{t})=\mathrm{A}+\mathrm{C} \exp \{-\exp [-\mathrm{B} \times(\mathrm{t}-\mathrm{M})]\}$ : the maximum specific growth rate $\mu$ max, the final multiplication of yEND, and tlag time lag.

\subsection{Preparation of Model Sausages}

White sausages were prepared under laboratory conditions from pork meat, curing salt, salt, water, spices, and extract of $C$. incanus $(6 \mathrm{~mL} / 100 \mathrm{~g}$ of meat) with either lycopene or betanin dyes (in the amount of $0.2 \mathrm{~g}$ of dye $/ 100 \mathrm{~g}$ of meat). Pork of different classes (I-lean, up to $15 \%$ fat; and II-medium fat, $16-20 \%$ ) was cut into cubes $(2 \pm 1 \mathrm{~cm})$. Spices, extracts, salt, and curing salt were added, depending on the type of sausage (Table 1). A curing time of $24 \mathrm{~h}$ was used, after which the meat was ground using a ZELMER meat grinder, stuffed into natural casings, and steamed until the temperature in the thermal center reached $74^{\circ} \mathrm{C}$. After cooling, the sausages were vacuum-packed and stored in a refrigerator for 28 days at $2 \pm 1{ }^{\circ} \mathrm{C}$. Four variants of sausages were prepared, differing in additives: CS — control sausage, containing pork meat and salt; NS — sausage containing pork meat, water, and a mixture containing salt and nitrates; CLS - sausage made of pork meat with $50 \%$ less nitrate content, with the addition of $C$. incanus herb extract and enriched with lycopene dye; CBS-sausage made of pork meat with 50\% less nitrate content, with the addition of $C$. incanus herb extract and beetroot coloring.

Table 1. Content of additives in the sausage variants.

\begin{tabular}{|c|c|c|c|c|c|}
\hline Ingredients & & CS & NS & CLS & CBS \\
\hline Water & \multirow{2}{*}{$\begin{array}{c}\mathrm{mL} / \\
100 \mathrm{~g}\end{array}$} & 20 & 20 & 14 & 14 \\
\hline $\begin{array}{c}\text { Cistus incanus } \\
\text { Extract }\end{array}$ & & - & - & 6 & 6 \\
\hline Salt & \multirow{6}{*}{$\begin{array}{c}\mathrm{g} / \\
100 \mathrm{~g}\end{array}$} & 1.8 & - & 0.9 & 0.9 \\
\hline Curing salt & & - & 1.8 & 0.9 & 0.9 \\
\hline Pepper & & 0.2 & 0.4 & 0.4 & 0.4 \\
\hline Granulated garlic & & 0.4 & 0.4 & 0.4 & 0.4 \\
\hline Beetroot powder & & - & - & - & 0.2 \\
\hline Lycopene powder & & - & - & 0.2 & - \\
\hline
\end{tabular}

\subsubsection{TBARS Index}

Samples of the four types of sausages were analyzed after $0,7,14,21$, and 28 days of storage. The stability of lipids was evaluated by determining the TBARS index, according to a modified method [24]. The sausages were ground in a ZELMER 686 mincer with a mesh of $8 \mathrm{~mm}$ holes. Next, $5.0 \mathrm{~g}$ of each sample was weighed and transferred to a distilled flask, to which $75 \mathrm{~mL}$ distilled water and $7 \mathrm{~mL}$ of $3 \mathrm{~N}$ hydrochloric acid were added. Steam distillation was performed to obtain $20 \mathrm{~mL}$ of distillate. Next, $3 \mathrm{~mL}$ of TBA solution was added and the samples were heated in a water bath under reflux coolers for $30 \mathrm{~min}$. The solutions were cooled, and the absorbance was measured at $\lambda=530 \mathrm{~nm}$. The concentration of malondialdehyde was calculated from the prepared standard curve.

\subsubsection{Color Determination}

The color of the sausage samples was measured after $0,7,14,21,28$ days of storage using the CIELa* $b^{*}$ method, where the L parameter measures the brightness on a range from 0 to 100, positive values for $\mathrm{a}^{*}$ indicate red color, negative values for $\mathrm{a}^{*}$ show green color, positive values for $\mathrm{b}^{*}$ show yellow color, and negative values for $\mathrm{b}^{*}$ indicate blue color. Spectrophotometric measurements were performed using a Konica Minolta Chroma Meter CR-400 (aperture $8 \mathrm{~mm}$ ) colorimeter (Japan). The $\Delta \mathrm{E}$ indices was calculated from the measurements, according to the formula: 


$$
\Delta E=\sqrt{(\Delta \boldsymbol{a})^{2}+(\Delta \boldsymbol{b})^{2}+(\Delta \boldsymbol{L})^{2}}
$$

\subsubsection{Microbiological Analysis}

Microbiological analysis of the sausages was performed after 0, 3, 5, 7, 14, 21, 28 days of storage. The tests were conducted in accordance with ISO 6887-2-2017 [25]. The total numbers of psychrotrophs and mesophiles were determined after incubation at $6{ }^{\circ} \mathrm{C}$ for 10 days and at $30^{\circ} \mathrm{C}$ for $72 \mathrm{~h}$ by counting bacterial colonies on agar plates (PCA, Merck). Enterobacteriaceae were also incubated at $30^{\circ} \mathrm{C}$ for $24 \mathrm{~h}$ on plates with growth medium (VRBG, Merck). Lactic acid bacteria were incubated at $30^{\circ} \mathrm{C}$ for $72 \mathrm{~h}$ on plates with MRS medium (Merck).

\subsubsection{Sensory Analysis}

Sensory analysis of the sausage variants was conducted by a selected panel of 10 people. The panel rated the taste, odor, color, and appearance of the meat products on a hedonic 5-point scale, where: 5 = extremely desirable, $4=$ desirable, $3=$ neither desirable nor undesirable, 2 = undesirable, 1 = extremely undesirable. Sensory analysis of the sausages was performed at time 0 , then after $5,14,21$, and 28 days. The taste of the meat products was evaluated at time 0 and 5 days after production.

\subsubsection{Statistical Analysis}

All assay variants were performed in triplicate. Excel 2010 was used to calculate the means and standard deviations of the samples. Analysis of variance (one-way ANOVA) was performed using STATISTICA 13.1. software. Tukey's honestly significant differences (HSD) post hoc test was used to determine differences between variables $(p<0.05)$.

\section{Results and Discussion}

\subsection{Antioxidant Activity C. incanus Extract and Dyes}

All the plant materials showed free radical scavenging ability (Table 2). The C. incanus extract, the beetroot extract, and the $C$. incanus extract in combination with beetroot and lycopene, had higher antioxidant activity than the lycopene extract, which showed the lowest activity. Higher values for free radical scavenging capacity were observed using the ABTS method than with DPPH. These differences were due to the properties of the reagents. ABTS interacts in both hydrophilic and lipophilic systems, whereas DPPH interacts in hydrophobic systems [26]. Previous studies have reported similar results to those produced in our study. Sayah [27] also observed strong antioxidant activity by Cistus extract. As in our study, Koubaier et al. [28] observed high antioxidant activity by beetroot extract. Many studies have shown that lycopene is a potent antioxidant and neutralizes free radicals. Its activity is increased by vitamins $C$ and $E$ as well as by $\beta$ carotene [29-31].

Table 2. Antioxidant activity measured by DPPH and ABTS methods (mean $\pm \mathrm{SD}, \mathrm{n}=3$ ).

\begin{tabular}{ccc}
\hline & DPPH & ABTS \\
\cline { 2 - 3 } & \multicolumn{2}{c}{$\mathrm{mg}$ Trolox $/ 100 \mathrm{~mL}$} \\
\hline C & $24.7 \pm 0.6^{\mathrm{a}}$ & $33.2 \pm 0.4^{\mathrm{a}}$ \\
\hline L & $18.5 \pm 0.3^{\mathrm{c}}$ & $21.4 \pm 0.2^{\mathrm{c}}$ \\
\hline B & $25.6 \pm 0.5^{\mathrm{a}}$ & $31.3 \pm 0.3^{\mathrm{b}}$ \\
\hline C $\mathbf{C}$ & $23.8 \pm 0.5^{\mathrm{b}}$ & $33.3 \pm 0.5^{\mathrm{a}}$ \\
\hline C B & $24.3 \pm 0.1^{\mathrm{a}, \mathrm{b}}$ & $32.4 \pm 0.5^{\mathrm{a}}$ \\
\hline
\end{tabular}

C, Cistus incanus extract; L, lycopene; B, betanin; C L, Cistus incanus extract with lycopene; C B, Cistus incanus with betanin; ${ }^{\mathrm{a}}, \mathrm{b}, \mathrm{c}$, significant differences between types of extracts $(p<0.05)$. 


\subsection{Polyphenolic Extract Composition}

The total polyphenolic content in the extract of $C$. incanus was $1642.98 \mathrm{mg}$ GAE/L of extract. Table 3 presents the qualitative composition of the aqueous extract of $C$. incanus. Forty-three compounds were identified. The largest groups in order of concentration were flavonols $(39.5 \%)>$ tannins $(13.95 \%)>$ benzoic acid derivatives $(13.95 \%)>$ procyanidins $(11.63 \%)>$ hydroxycinnamic acid derivatives, phenolic acids $(4.65 \%)$, flavan-3-ols and other compounds. The $C$. incanus extract contained quinic and succinic phenolic acids. The following tannins were identified: punicalin isomer, cornusin B, HHDP (hexahydroxydiphenylglucose isomer), galloyl- HHDP-glucoside, punicalagin isomer, and punicalagin-gallate isomer. The flavonoids included myricetin derivatives, kaempferol derivatives, and quercetin. The extract contained the following procyanidins: gallocatechin, gallic acid, gallocatechin trimer, gallocatechin-( $4 \alpha-8)$-catechin, epicatechin-3-gallate, catechin and epicatechin.

Table 3. Polyphenols in aqueous extract of C. incanus identified using the MS method.

\begin{tabular}{|c|c|c|c|c|}
\hline $\begin{array}{l}\text { Retention } \\
\text { Time [min] }\end{array}$ & $\lambda \max$ & $m / z$ & $\mathrm{~ms}^{2}$ & Compound \\
\hline 2.20 & $345 ; 378$ & 781 & $601 ; 301$ & Punicalin isomer \\
\hline 2.21 & $255 ; 360$ & 1085 & $783 ; 451 ; 425 ; 301$ & Cornusin B \\
\hline 2.29 & $218 ; 245$ & 195 & 138 & Caffeine \\
\hline 2.37 & $250 ; 277$ & 191 & $173 ; 171$ & Quinic acid \\
\hline 2.96 & $256 ; 280$ & 331 & $481 ; 673 ; 779 ; 963$ & Galloyl glucose \\
\hline 2.96 & 279 & 913 & 305 & Gallocatechin trimer \\
\hline 3.00 & $238 ; 376$ & 481 & $301 ; 275 ; 257 ; 229$ & Hexahydroxydiphenyl-glucose isomer (HHDP) \\
\hline 3.02 & 260 & 117 & 73 & Succinic acid \\
\hline 3.49 & 265 & 331 & $225 ; 481 ; 897$ & Galloyl glucose \\
\hline 3.88 & 270 & 343 & $191 ; 169 ; 125$ & 3-O-galloquinic acid \\
\hline 3.93 & $213 ; 261$ & 169 & 125 & Gallic acid \\
\hline 4.01 & 275 & 609 & $591 ; 483 ; 441 ; 423 ; 305$ & 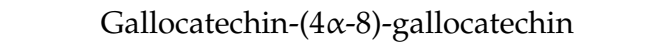 \\
\hline 4.34 & 280 & 483 & 169 & Digalloyl glucose \\
\hline 4.50 & $260 ; 379$ & 781 & $601 ; 301$ & Punicalin isomer \\
\hline 5.20 & $255 ; 275$ & 913 & 305 & Gallocatechin trimer \\
\hline 5.21 & $260 ; 285$ & 609 & $423 ; 305 ; 483 ; 591$ & 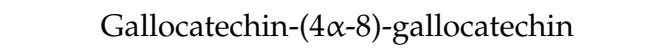 \\
\hline 5.30 & 265 & 331 & $225 ; 481 ; 897$ & Galloyl glucose \\
\hline 5.47 & $256 ; 288$ & 913 & 305 & Gallocatechin trimer \\
\hline 5.84 & $259 ; 288$ & 593 & $575 ; 467 ; 425 ; 407 ; 289$ & Gallocatechin- $(4 \alpha-8)$-catechin \\
\hline 6.09 & 278 & 483 & 169 & Digalloyl glucose \\
\hline 6.25 & $240 ; 267$ & 153 & 109 & Protocatechuic acid \\
\hline 6.38 & $260 ; 273 ; 299$ & 305 & $287 ; 261 ; 221 ; 219 ; 179$ & Gallocatechin \\
\hline 6.91 & $260 ; 278$ & 609 & $423 ; 305 ; 483 ; 591$ & 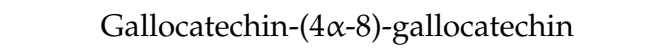 \\
\hline 7.04 & $305 ; 345$ & 593 & $447 ; 285$ & Kaempferol-3-rutinoside \\
\hline 7.10 & 310 & 355 & 195 & Cis or trans ferulic acid hexoside \\
\hline 8.27 & 238 & 633 & 301; & Galloyl-HHDP-glucoside \\
\hline 9.01 & $320 ; 365$ & 1083 & $781 ; 601 ; 301$ & Punicalagin isomer \\
\hline 9.01 & $255 ; 370$ & 1085 & $783 ; 451 ; 425 ; 301$ & Cornusin B \\
\hline 9.45 & 376 & 1251 & $1207 ; 1082 ; 781 ; 601 ; 301$ & Punicalagin-gallate isomer \\
\hline 9.53 & 278 & 289 & $271 ; 245 ; 205 ; 179$ & Catechin \\
\hline 9.79 & $249 ; 333$ & 351 & & $\mathrm{Ni}$ \\
\hline 9.90 & $320 ; 365$ & 1083 & $781 ; 601 ; 301$ & Punicalagin isomer \\
\hline 9.90 & $255 ; 370$ & 1085 & $783 ; 451 ; 425 ; 301$ & Cornusin B \\
\hline 9.90 & 278 & 633 & 301 & Galloyl-HHDP-glucoside \\
\hline 10.10 & $265 ; 289$ & 305 & $287 ; 261 ; 221 ; 219 ; 179$ & Gallocatechin \\
\hline 10.37 & 238 & 481 & $301 ; 275 ; 257 ; 229$ & Hexahydroxydiphenyl-glucose isomer(HHDP) \\
\hline 10.57 & $267 ; 286$ & 179 & 135 & Caffeic acid \\
\hline 11.07 & $256 ; 375$ & 461 & 415 & Diosmetin 8-C-glucoside \\
\hline 11.22 & $258 ; 376$ & 1251 & $1207 ; 1082 ; 781 ; 601 ; 301$ & Punicalagin-gallate isomer \\
\hline 11.24 & $260 ; 350$ & 625 & 316 & Myricetin derivative \\
\hline 11.29 & $238 ; 377$ & 481 & $301 ; 275 ; 257 ; 229$ & Hexahydroxydiphenyl-glucose isomer(HHDP) \\
\hline
\end{tabular}


Table 3. Cont.

\begin{tabular}{|c|c|c|c|c|}
\hline $\begin{array}{l}\text { Retention } \\
\text { Time [min] }\end{array}$ & $\lambda \max$ & $m / z$ & $\mathrm{~ms}^{2}$ & Compound \\
\hline 11.70 & $260 ; 349$ & 449 & 447 & Myricetin-pentoside \\
\hline 12.01 & $256 ; 350$ & 433 & $301 ; 271$ & Myricetin derivative \\
\hline 12.05 & $276 ; 289$ & 441 & 169 & Epicatechin-3-gallate \\
\hline 13.29 & 285 & 163 & 119 & $p$-Coumaric acid \\
\hline 13.44 & 355 & 475 & 285 & Kaempferoldimethyletherhexoside \\
\hline 13.75 & 280 & 483 & 169 & Digalloyl glucose \\
\hline 13.89 & 345 & 625 & 316 & Myricetin derivative \\
\hline 14.11 & 351 & 479 & $317 ; 289 ; 271 ; 179,151$ & Myricetin-galactoside \\
\hline 14.18 & $266 ; 278$ & 137 & 93 & Salicylic acid \\
\hline 15.88 & $260 ; 351$ & 449 & 447 & Myricetin-O-xyloside \\
\hline 16.17 & 279 & 913 & 305 & Gallocatechin trimer \\
\hline 16.21 & $260 ; 351$ & 479 & $317 ; 289 ; 271 ; 179,151$ & Myricetin-3-O-galactoside \\
\hline 16.21 & $260 ; 355$ & 463 & $317 ; 289 ; 271 ; 179 ; 151$ & Myricetin-rhamnoside \\
\hline 16.31 & $255 ; 345$ & 609 & 463 & Quercetin rhamno glucoside \\
\hline 16.71 & $260 ; 355$ & 625 & $317 ; 271 ; 179 ; 151$ & Myricetin-rutinoside \\
\hline 18.21 & 270 & 289 & $245 ; 205 ; 271$ & Epicatechin \\
\hline 18.42 & $255 ; 345$ & 447 & $301 ; 271 ; 255 ; 179$ & Quercetin-3-O-rhamnoside \\
\hline 18.58 & $258 ; 350$ & 433 & 301 & Quercetin derivative \\
\hline 18.75 & $260 ; 345$ & 593 & 285 & Keampferol- rhamno-glucoside \\
\hline 19.62 & $267 ; 350$ & 447 & 285 & Kaempferol-3-O-glucoside \\
\hline 19.66 & $281 ; 296$ & 483 & 169 & Digalloyl glucose \\
\hline 23.85 & 355 & 625 & $607 ; 479 ; 317 ; 179$ & Myricetin rhamno-glucoside \\
\hline 24.70 & $253 ; 284$ & 301 & 151 & Quercetin \\
\hline
\end{tabular}

HHDP, hexahydroxydiphenyl-glucose isomer, $\mathrm{Ni}$, not identified.

Barrajón-Catalán et al. [32] and Riehle et al. [33] identified flavan-3-ols in C. incanus extracts, and found various flavanols, especially the compounds myricetin, quercetin, and kaempferol. Wittpahl et al. [19] also identified tannins: punicalin isomers, punicalagin isomers, and cornusin B.

\subsection{Antibacterial Activity of C. incanus Extract and Dyes}

The culture method was used to study the impact of the $C$. incanus extract and dyes on the activity of selected Gram negative and Gram positive bacteria. Cistus incanus was applied in concentrations of $0.5 \%, 1.0 \%$, and $2.0 \%$ with $1 \%$ of both lycopene and betanin dyes, or with $1 \%$ of either dye. The numbers of microorganisms were determined by the average colony count method on three plates after a specific seeding time. Based on the results, the growth parameters were calculated using the Gompertz function. After fitting the results, a correlation coefficient of between 0.962 and 0.999 was obtained. We calculated the following parameters: proper growth rate ( $\mu$ max), duration of lag-phase (tLag), final number of cells (yEnd). The dye extracts had different effects on the selected bacteria. The results are presented in Tables 4 and 5. It was observed that as the concentration of C. incanus extract increased, the multiplication rate of the tested bacteria decreased. The application of $C$. incanus extract in concentrations of $0.5 \%, 1.0 \%$, and $2.0 \%$ decreased the growth rate to a statistically significant degree. The use of the extract resulted in a lower growth rate and lag time of the bacterial phase compared to the control samples. The C. incanus extract interacted against the Gram positive bacteria better than against the Gram negative bacteria. 
Table 4. Effects of $C$. incanus extract and dyes on the maximum specific growth rate ( $\mu$ max) of bacteria $($ mean $\pm S D, n=3)$.

\begin{tabular}{|c|c|c|c|c|c|c|}
\hline & Escherichia coli & $\begin{array}{l}\text { Pseudomonas } \\
\text { fragi }\end{array}$ & $\begin{array}{l}\text { Salmonella } \\
\text { enterica }\end{array}$ & $\begin{array}{l}\text { Brochothrix } \\
\text { thermosphacta }\end{array}$ & $\begin{array}{c}\text { Latilactobacillus } \\
\text { sakei }\end{array}$ & $\begin{array}{c}\text { Listeria } \\
\text { monocytogenes }\end{array}$ \\
\hline & \multicolumn{6}{|c|}{$\mu \max \left[h^{-1}\right]$} \\
\hline Control & $0.844 \pm 0.021^{\mathrm{a} \mathrm{A}}$ & $0.438 \pm 0.010^{\mathrm{aE}}$ & $0.630 \pm 0.035^{\mathrm{aC}}$ & $0.542 \pm 0.013^{\mathrm{aD}}$ & $0.547 \pm 0.007^{\mathrm{a} \mathrm{D}}$ & $0.819 \pm 0.014^{\mathrm{aA}}$ \\
\hline C 0.5 & $0.726 \pm 0.032^{\mathrm{bB}}$ & $0.407 \pm 0.005^{\mathrm{bE}}$ & $0.593 \pm 0.018^{\mathrm{aD}}$ & $0.412 \pm 0.005^{\mathrm{eE}}$ & $0.267 \pm 0.014^{\mathrm{eF}}$ & $0.844 \pm 0.025^{\mathrm{aA}}$ \\
\hline C 1.0 & $0.621 \pm 0.035^{\mathrm{cB}}$ & $0.405 \pm 0.011^{\mathrm{bD}}$ & $0.483 \pm 0.011^{\mathrm{cD}}$ & $0.312 \pm 0.015^{\mathrm{gE}}$ & $0.263 \pm 0.005^{\mathrm{eF}}$ & $0.721 \pm 0.008^{\mathrm{bA}}$ \\
\hline C 2.0 & $0.432 \pm 0.024^{\mathrm{eB}}$ & $0.395 \pm 0.013^{b c}$ & $0.469 \pm 0.023^{\mathrm{cB}}$ & $0.292 \pm 0.010^{g D}$ & $0.260 \pm 0.022^{\mathrm{eD}}$ & $0.582 \pm 0.007^{\mathrm{cA}}$ \\
\hline $\mathrm{L}$ & $0.451 \pm 0.005^{\mathrm{eB}}$ & $0.410 \pm 0.021^{\mathrm{a}, \mathrm{b} b}$ & $0.444 \pm 0.007^{\mathrm{c}, \mathrm{d} B}$ & $0.293 \pm 0.027^{g C}$ & $0.500 \pm 0.021^{\mathrm{bA}}$ & $0.429 \pm 0.014^{\mathrm{eB}}$ \\
\hline B & $0.560 \pm 0.017^{\mathrm{d} A}$ & $0.351 \pm 0.015^{\mathrm{cC}}$ & $0.432 \pm 0.021^{\mathrm{c}, \mathrm{d} B}$ & $0.409 \pm 0.017^{\mathrm{eB}}$ & $0.512 \pm 0.004^{\mathrm{bA}}$ & $0.567 \pm 0.017^{\mathrm{cA}}$ \\
\hline C $0.5 \mathrm{~L}$ & $0.825 \pm 0.034^{\text {a A }}$ & $0.455 \pm 0.021^{\mathrm{aE}}$ & $0.613 \pm 0.017^{a C}$ & $0.520 \pm 0.009^{\mathrm{aD}}$ & $0.277 \pm 0.013^{e F}$ & $0.493 \pm 0.013^{\mathrm{dE}}$ \\
\hline C $1.0 \mathrm{~L}$ & $0.810 \pm 0.021^{\mathrm{a} \mathrm{A}}$ & $0.445 \pm 0.003^{\mathrm{a} \mathrm{E}}$ & $0.583 \pm 0.015^{\mathrm{a}, \mathrm{b} \mathrm{D}}$ & $0.454 \pm 0.010^{\mathrm{dE}}$ & $0.289 \pm 0.008^{\mathrm{e} G}$ & $0.391 \pm 0.025^{\mathrm{e}, \mathrm{fF}}$ \\
\hline C $2.0 \mathrm{~L}$ & $0.803 \pm 0.019^{\mathrm{a} A}$ & $0.426 \pm 0.010^{a, b} \mathrm{E}$ & $0.573 \pm 0.021^{\mathrm{a}, \mathrm{bD}}$ & $0.381 \pm 0.017^{\mathrm{e}, \mathrm{fF}}$ & $0.269 \pm 0.019^{\mathrm{eG}}$ & $0.413 \pm 0.011^{\mathrm{e}, \mathrm{fE}}$ \\
\hline C $0.5 \mathrm{~B}$ & $0.795 \pm 0.024^{\mathrm{aA}}$ & $0.435 \pm 0.015^{\mathrm{a} \mathrm{D}}$ & $0.591 \pm 0.009^{\mathrm{a} \mathrm{C}}$ & $0.534 \pm 0.015^{\mathrm{aC}}$ & $0.340 \pm 0.011^{\mathrm{cE}}$ & $0.456 \pm 0.025^{\mathrm{d}, \mathrm{eD}}$ \\
\hline C $1.0 \mathrm{~B}$ & $0.780 \pm 0.021^{a, b ~ A ~}$ & $0.345 \pm 0.025^{\mathrm{cE}}$ & $0.564 \pm 0.028^{a, b C}$ & $0.505 \pm 0.006^{b C}$ & $0.315 \pm 0.007^{\mathrm{dE}}$ & $0.434 \pm 0.014^{\mathrm{eD}}$ \\
\hline C $2.0 \mathrm{~B}$ & $0.720 \pm 0.015^{\mathrm{bA}}$ & $0.266 \pm 0.004^{\mathrm{dE}}$ & $0.500 \pm 0.010^{\mathrm{cC}}$ & $0.495 \pm 0.013^{b, c D}$ & $0.253 \pm 0.023^{\mathrm{eE}}$ & $0.437 \pm 0.008^{\mathrm{eD}}$ \\
\hline
\end{tabular}

C 0.5, Cistus incanus extract $0.5 \%$; C 1.0, Cistus incanus extract $1.0 \%$; C $2.0 \%$, Cistus incanus extract $2.0 \%$; L, lycopene; B, betanin; C $0.5 \mathrm{~L}$, Cistus incanus extract $0.5 \%$ with lycopene; C $1.0 \mathrm{~L}$, Cistus incanus extract $1.0 \%$ with lycopene; C $2.0 \mathrm{~L}$, Cistus incanus extract $2.0 \%$ with lycopene; C 0.5 B, Cistus incanus extract $0.5 \%$ with betanin; C 1.0 B, Cistus incanus extract $1.0 \%$ with betanin; C 2.0 B, Cistus incanus extract $2.0 \%$ with betanin; ${ }^{\mathrm{a}-\mathrm{g}}$, significant differences between variants against the same bacterial strain $(p<0.05)$. ${ }^{\mathrm{A}-\mathrm{G}}$, significant differences between types of bacterial strain for the same variant $(p<0.05)$. The colors indicate values from the highest (red) through medium (yellow) to the lowest (green).

The application of $0.5 \%$ C. incanus extract contributed to a $51.2 \%$ reduction in the growth rate of L. sakei. However, against Gram negative E. coli bacteria, 2.0\% C. incanus extract slowed the growth by $48.8 \%$ compared to the control. The weakest effect was observed in the case of P. fragi. Both lycopene and beetroot pigments slowed the growth rate of Gram positive bacteria, with the best results against $B$. thermosphacta $(45.9 \%$ reduction in growth rate compared to the control). Lycopene was stronger against Gram negative bacteria, reducing the growth rate of $E$. coli by $46.6 \%$. No synergistic effect between the $C$. incanus extract and the lycopene and betanin dyes was observed at the tested concentrations. No statistically significant changes were observed for Gram negative E. coli and S. enterica. Cistus incanus extract at concentrations of 1.0 and 2.0 in combination with betanin slowed the specific growth rate of $P$. fragi the most. The extract of $C$. incanus with both lycopene and betanin slowed the growth rate of the Gram positive bacteria L. sakei and L. monocytogenes best. Against $B$. thermosphacta, $2.0 \%$ extract of $C$. incanus in combination with lycopene was the most effective.

Another measured parameter was the duration of the lag phase. As the concentration of $C$. incanus extract was increased, the duration of lag increased compared to the control sample. For Gram negative bacteria, lag was best extended by $C$. incanus at a concentration of $2.0 \%$ against $P$. fragi, and for Gram positive bacteria by B. thermosphacta and L. sakei. Lycopene and beetroot dye extracts prolonged lag phase duration the most, by $202.7 \%$ and $205.3 \%$, respectively, against $L$. sakei. The dyes also increased the lag time of $L$. monocytogenes by $70 \%$. Against Gram negative bacteria, the dyes had the strongest effect on the lag phase of S. enterica, extending its duration by $136.3 \%$ in the case of lycopene and by $128.0 \%$ for beet. When $C$. incanus extract was used at three concentrations together with dyes, $1.0 \%$ and $2.0 \%$ C. incanus with lycopene and $2.0 \%$ C. incanus with beetroot had the best effects against B. thermosphacta and P. fragi, respectively. The differences in the final bacterial proliferation (yEnd) values were not statistically significant.

According to the literature, C. incanus extracts inhibit the growth of Staphylococcus aureus and Escherichia coli bacteria [19,34]. Rajnbar [35] studied the antimicrobial activity of lycopene oleoresin against the pathogens Pseudomonas aeruginosa, Escherichia coli, Staphylococcus ureuse, Salmonella typhimurium, Listeria monocytogenes, Bacillus cereus, and Bacillus licheniformis. It was found that tomato peel oleoresin containing $2 \%$ lycopene can inhibit and restrain both Gram negative the Gram positive bacteria. There are no data in the literature on the effects of $C$. incanus, lycopene and beetroot extracts on microbial growth parameters. 
Table 5. Effects of $C$. incanus extract and dyes on lag time of bacteria (mean $\pm S D, n=3$ ).

\begin{tabular}{|c|c|c|c|c|c|c|}
\hline & Escherichia coli & $\begin{array}{c}\text { Pseudomonas } \\
\text { fragi }\end{array}$ & $\begin{array}{l}\text { Salmonella } \\
\text { enterica }\end{array}$ & $\begin{array}{c}\text { Brochothrix } \\
\text { thermosphacta }\end{array}$ & $\begin{array}{l}\text { Latilactobacillus } \\
\text { sakei }\end{array}$ & $\begin{array}{c}\text { Listeria } \\
\text { monocytogenes }\end{array}$ \\
\hline & \multicolumn{6}{|c|}{ tLag $\left[h^{-1}\right]$} \\
\hline Control & $2.59 \pm 0.05^{\mathrm{cC}}$ & $4.67 \pm 0.31^{\mathrm{d} A}$ & $1.82 \pm 0.13^{\mathrm{dD}}$ & $3.17 \pm 0.22 \mathrm{gB}$ & $3.01 \pm 0.07 \mathrm{~g} \mathrm{~B}$ & $3.38 \pm 0.25^{\mathrm{fB}}$ \\
\hline C 0.5 & $2.52 \pm 0.07^{\mathrm{cD}}$ & $5.33 \pm 0.15^{\mathrm{c} \mathrm{A}}$ & $1.79 \pm 0.15^{\mathrm{dE}}$ & $4.51 \pm 0.11^{\mathrm{fB}}$ & $3.37 \pm 0.15^{f C}$ & $4.29 \pm 0.09^{\mathrm{cB}}$ \\
\hline C 1.0 & $3.09 \pm 0.09^{b c}$ & $5.50 \pm 0.22^{\mathrm{cA}}$ & $2.73 \pm 0.11^{\mathrm{bD}}$ & $5.15 \pm 0.07^{\mathrm{e} A}$ & $5.38 \pm 0.11^{d A}$ & $4.39 \pm 0.05^{\mathrm{cB}}$ \\
\hline C 2.0 & $3.68 \pm 0.15^{\mathrm{aE}}$ & $6.05 \pm 0.15^{\mathrm{bB}}$ & $2.80 \pm 0.16^{b F}$ & $7.18 \pm 0.13^{c A}$ & $6.87 \pm 0.25^{\mathrm{bB}}$ & $4.94 \pm 0.12^{\mathrm{bD}}$ \\
\hline $\mathrm{L}$ & $3.53 \pm 0.03^{a, b F}$ & $4.74 \pm 0.20^{\mathrm{d} E}$ & $4.30 \pm 0.13^{a \mathrm{E}}$ & $3.82 \pm 0.08 \mathrm{gF}^{2}$ & $9.11 \pm 0.15^{\text {a A }}$ & $5.73 \pm 0.03^{\mathrm{a} \mathrm{D}}$ \\
\hline B & $3.42 \pm 0.09^{a, b F}$ & $4.65 \pm 0.15^{\mathrm{d} E}$ & $4.15 \pm 0.08^{a \mathrm{E}}$ & $5.65 \pm 0.15^{\mathrm{d} D}$ & $9.19 \pm 0.09^{\text {a } A}$ & $5.65 \pm 0.07^{\mathrm{a} D}$ \\
\hline C $0.5 \mathrm{~L}$ & $2.84 \pm 0.16^{\mathrm{bD}}$ & $4.85 \pm 0.08^{\mathrm{dB}}$ & $1.92 \pm 0.20^{\mathrm{dE}}$ & $3.47 \pm 0.11^{\mathrm{gC}}$ & $5.82 \pm 0.11^{\mathrm{cA}}$ & $3.76 \pm 0.08^{\mathrm{eC}}$ \\
\hline C $1.0 \mathrm{~L}$ & $3.15 \pm 0.11^{b E}$ & $6.23 \pm 0.05^{\mathrm{b} \mathrm{B}}$ & $2.48 \pm 0.11^{\mathrm{cF}}$ & $7.45 \pm 0.07^{\mathrm{b} \mathrm{A}}$ & $6.02 \pm 0.09^{\mathrm{cB}}$ & $3.80 \pm 0.12^{\mathrm{e} E}$ \\
\hline C $2.0 \mathrm{~L}$ & $3.57 \pm 0.15^{\mathrm{a} \mathrm{E}}$ & $6.78 \pm 0.31^{\mathrm{a} \mathrm{B}}$ & $2.81 \pm 0.09 \mathrm{bF}$ & $7.47 \pm 0.11^{\mathrm{b} \mathrm{A}}$ & $6.06 \pm 0.09^{\mathrm{cB}}$ & $4.26 \pm 0.13^{\mathrm{cD}}$ \\
\hline C $0.5 \mathrm{~B}$ & $2.95 \pm 0.07^{b C}$ & $4.70 \pm 0.16^{\mathrm{d} A}$ & $1.85 \pm 0.08^{\mathrm{dD}}$ & $3.49 \pm 0.05 \mathrm{gB}$ & $3.50 \pm 0.03^{f B}$ & $3.28 \pm 0.15^{\mathrm{fB}}$ \\
\hline C $1.0 \mathrm{~B}$ & $3.45 \pm 0.09^{a, b c}$ & $4.78 \pm 0.13^{\mathrm{dB}}$ & $2.65 \pm 0.04^{b D}$ & $5.71 \pm 0.11^{\mathrm{d} A}$ & $4.59 \pm 0.21^{\mathrm{e} B}$ & $3.88 \pm 0.13^{\mathrm{eC}}$ \\
\hline C $2.0 \mathrm{~B}$ & $3.73 \pm 0.10^{\text {a F }}$ & $5.28 \pm 0.07^{\mathrm{CD}}$ & $2.60 \pm 0.09^{b, c ~ G}$ & $8.11 \pm 0.21^{\text {a A }}$ & $4.75 \pm 0.05^{\mathrm{eE}}$ & $4.05 \pm 0.01^{\mathrm{d} E}$ \\
\hline
\end{tabular}

C 0.5, Cistus incanus extract 0.5\%; C 1.0, Cistus incanus extract 1.0\%; C 2.0\%, Cistus incanus extract 2.0\%; L, lycopene; B, betanin; C 0.5 L, Cistus incanus extract $0.5 \%$ with lycopene; C $1.0 \mathrm{~L}$, Cistus incanus extract $1.0 \%$ with lycopene; C $2.0 \mathrm{~L}$, Cistus incanus extract $2.0 \%$ with lycopene; C 0.5 B, Cistus incanus extract $0.5 \%$ with betanin; C 1.0 B, Cistus incanus extract $1.0 \%$ with betanin; C 2.0 B, Cistus incanus extract $2.0 \%$ with betanin; ${ }^{a-g}$, significant differences between types of extracts against the same bacterial strain $(p<0.05)$. ${ }^{\mathrm{A}-\mathrm{G}}$, significantly differences between types of bacterial strain the same extract $(p<0.05)$. The colors indicate values from the highest (red) through medium (yellow) to the lowest (green).

The antimicrobial activity is not due to the total amount of polyphenols but to the chemical composition of the extracts [36]. Numerous flavonoids: flavan-3-ols, flavonols and tannins were identified in the extract of Cistus incanus we studied. According to literature data, flavonoids are characterized by strong antimicrobial properties. Quercetin, myricetin, kaempferol and their derivatives, as well as flavones, flavanones, flavan-3-ols and chalcones found in plants inhibit bacterial growth [37]. In contrast, tannins have weak antimicrobial activity against Gram negative bacteria [38]. The punicalagin isomers found in our extract are known for their antioxidant, antibacterial and antiviral properties. The available studies checked the activity of punicalagin against $S$. aureus by agar diffusion method. Based on the results, punicalagin showed good activity against Staphylococcus aureus. Moreover, it had a significant inhibitory effect on S. aureus biofilm formation [39]. In a study by Kuchta et al., who also tested the aqueous extract of Cistus incanus for antimicrobial activity, it was observed that the aqueous extract of $C$. incanus showed no activity against Gram negative bacteria [40]. The previous studies also confirm the good activity of aqueous extracts of Cistus incanus against Gram positive and to a lesser extent against Gram negative [19,41]. Our study is in agreement with literature data which indicate that Gram positive bacteria are more sensitive to polyphenols than Gram negative bacteria [42]. Although the walls of Gram negative bacteria are covered with a lipophilic outer membrane that provides protection, and this contributes to a lower permeability of hydrophilic substances, the presence of polyphenols can disrupt the outer membrane of Gram negative bacteria, thereby increasing the permeability of the membrane $[43,44]$. To the best of our knowledge, the effect of $C$. incanus extract on bacterial growth parameters, proper growth rate and lag phase duration, has not been studied yet. Thus, further studies are required to investigate the mechanism of action of Cistus incanus extract and the bioactive compounds present in it.

\subsection{Effect of Polyphenolic Extract and Dyes on Lipid Oxidation in Pork Sausages}

Figure 1 shows the effects of $C$. incanus extract with lycopene and betanin dyes on lipid oxidation during cold storage of pork sausages. The content of malondialdehyde, which is one of the lipid oxidation products, varied during storage. The highest content was observed in the control sausage with salt (CS) and was $3.35 \mathrm{mg} / 100 \mathrm{~g}$ meat. However, in the other meat products the aldehyde content was several times lower. After the first week of storage, an increase in aldehyde content was observed in all the analyzed sausages. The highest increase was in sausages with salt and was $3.36 \mathrm{mg} / 100 \mathrm{~g}$ of meat. The lowest increase was observed in the case of sausages with C. incanus and lycopene. 
After two weeks, the malondialdehyde content of the salted sausages (CS) increased by $17.31 \%$ compared to the baseline sample. In the remaining samples, a slight increase was observed compared to samples after 7 days of storage, with the lowest increases for sausages with $C$. incanus extract and lycopene (CLS) and with $C$. incanus and betanin (CBS). This indicates that extracts in combination with dyes slow lipid oxidation processes. After three weeks of storage, the highest increase in malondialdehyde content (eight times higher) was recorded for sausages with nitrates (PS). Conversely, a significant increase was observed in the malondialdehyde content of sausages with $C$. incanus and betanin (as high as six times higher than in the initial samples). After four weeks of storage, the highest malondialdehyde concentration at $4.81 \mathrm{mg} / 100 \mathrm{~g}$ of meat was obtained for sausages with salt (CS). On the basis of these results, it can be concluded that the use of $C$. incanus extract in combination with beetroot and tomato peel colorant and a reduced amount of nitrates slows the process of lipid oxidation in stored pork sausages.

Zhang et al. [45] evaluated the effects of clove extract on the quality and fat oxidation of sausages. As in our study, they observed slowing of lipid oxidation processes and inhibited deterioration. Efenberger-Szmechtyk et al. [36] observed that the addition of leaf extracts of A. melanocarpa, C. superba, and C. mas to pork sausages stored in MAP inhibited lipid oxidation. The $C$. superba extract had the greatest effect on the MDA concentration in the sausages.

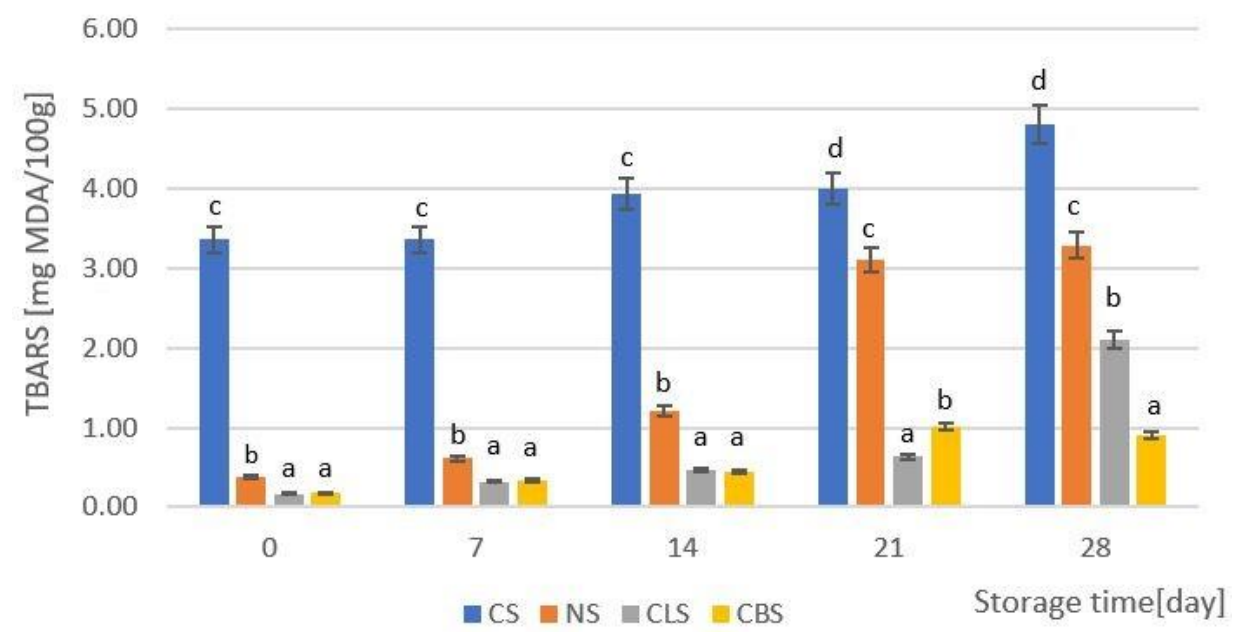

Figure 1. Effects of $C$. incanus extract with lycopene and betanin on lipid oxidation in vacuum packed pork sausages stored at $2 \pm 1{ }^{\circ} \mathrm{C}$. The results are expressed as mean $\pm \mathrm{SD}, \mathrm{n}=3$. CS, sausages with salt; NS, sausages with curing salt; CLS, sausages with $C$. incanus extract and lycopene; CBS, sausages with $C$. incanus extract and betanin; a-d, statistically differences between the sausage variants $(p<0.05)$.

\subsection{Effect of Polyphenolic Extract and Dyes on Sausage Color}

Color and color stability are important quality parameters for meat and meat products [46]. Table 6 shows the values for the parameters of brightness $L^{*}$, redness $a^{*}$, yellowness $\mathrm{b}^{*}$, and color stability $\Delta \mathrm{E}$ in pork sausages with and without the $C$. incanus extract and natural dyes. The sausages were initially characterized by brightness ranging from 60.01 to 64.82. The darkest sausage was the one with $C$. incanus extract and beetroot. The brightest was the sausage with salt. Similar brightness levels were recorded for all the sausages with the extract and dyes and reduced nitrate content. After one week of storage, the sausage with $C$. incanus extract and betanin (CBS) was still the darkest, while the sausage with NS nitrate was the lightest. After two weeks, the sausages with salt (CS) and nitrates (NS) were darker, as were the sausages with natural additives. However, after 3 weeks of storage, the sausages with salt and the sausages with nitrates darkened, while sausages with the extract and dyes became lighter. After four weeks of storage, the sausages with salt (CS) and curing salt (NS) were darker and the other sausages were lighter. 
The highest proportion of red color (parameter $\mathrm{a}^{*}$ ) was measured for sausages with a lower content of nitrates, with $C$. incanus extract and enriched with betanin (CBS). The share of red color was the lowest in the sausages with salt (CS) but was 50\% higher in the sausages with nitrates compared to the sausages without added nitrates. This confirms that curing salt fulfils its function and provides meat products with their characteristic pink color. At the same time, the addition of the extract and natural colorants increased the proportion of red color in the sausages. After the first week of storage, the greatest decrease in the content of red color of as much as 5\% was recorded for sausages with nitrates (NS). A reduction in parameter $\mathrm{a}^{*}$ was also observed in the sausages with $C$. incanus and betanin (CBS). After 7 days, the sausage with $C$. incanus and betanin extract (CBS) was still characterized by the highest proportion of red color. This trend continued throughout the 28-day storage period. After 14 days, the proportion of red color in the sausages with salt (CS) decreased by $9 \%$ in comparison to the color immediately after manufacture. The proportion of red increased in the remaining sausages. The greatest increase was observed for sausages enriched with lycopene dye (CLS). In the next stage of storage, the CBS sausages were characterized by the highest share of red color, which indicates that the addition of the $C$. incanus extract and enrichment with betanin increased and stabilized the share of red color in the sausages. The lowest proportions of red color after 3 and 4 weeks were in the CS and NS sausages.

In our previous study, which analyzed the effects of $C$. incanus extract on selected parameters of meat products, the plant extract positively increased the proportion of red color in sausages with reduced nitrates [47]. The $b^{*}$ parameter, indicating the proportion of yellow color, was initially highest in the sausages with CS salt and was maintained during storage. The lowest $b^{*}$ value was detected in sausages with nitrates (NS) and in sausages with C. incanus and beetroot (CBS). After 7 days of storage, an increase in the proportion of yellow color was observed in all the sausages. During storage, the $b^{*}$ value increased in all the analyzed sausages. The samples containing $C$. incanus extract and enriched with lycopene (CLS) and betanin (CBS) had a greater color difference $\Delta \mathrm{E}$ compared to the salt and nitrate samples. A similar result was obtained by Nowak et al. [48] using cherry and black currant leaf extracts as additives in sausages. Noticeable changes in color $\Delta \mathrm{E}>2$ were noticed for only one type of CBS sausage.

\subsection{Effect of Polyphenolic Extract and Dyes on Microbial Stability of Sausages}

The results of microbiological test on the vacuum-packed pork sausages made during the 28-day storage period are shown in Table 7. At the end of the storage time, the amounts of mesophilic bacteria, psychrotrophs, lactic acid bacteria, and Enterobacteriaceae in the pork sausages increased by statistically significant amounts. After production, the pork sausages containing the plant extract and colorants showed small amounts of the tested bacterial groups and there were no bacteria from the Enterobacteriaceae family. The smallest amount of mesophilic bacteria was recorded in the sausages containing lycopene and extract of Cistus incanus. During storage, the C. incanus extract and beetroot increased the bacterial count in the sausages. The greatest increase in the amount of psychrotroph bacteria was noticed after day 7 of storage. On days 7 and 14 of storage, the smallest number of Enterobacteriaceae was recorded for sausages with $C$. incanus extract with lycopene. In terms of the amount of psychrotrophs, the smallest significantly statistical differences $(p<0.05)$ were recorded on day 14 of storage. Further research is needed to test whether higher concentrations of the plant extract and dyes could inhibit the growth of the studied groups of bacteria. The reduced amount of nitrates in the sausages with $C$. incanus extract and dyes did not impair the microbiological quality of the meat products. 
Table 6. Effects of $C$. incanus extract with lycopene and betanin on color parameters $\left(\mathrm{L}^{*}, \mathrm{a}^{*}, \mathrm{~b}^{*}\right)$ of pork sausages stored in vacuum packaging at $2 \pm 1{ }^{\circ} \mathrm{C}($ mean $\pm \mathrm{SD}, \mathrm{n}=3)$.

\begin{tabular}{|c|c|c|c|c|c|}
\hline & & & Lightness L* & & \\
\hline $\begin{array}{l}\text { Day } \\
\text { CS } \\
\text { NS } \\
\text { CLS } \\
\text { CBS }\end{array}$ & $\begin{array}{c}0 \\
64.82 \pm 0.25^{\text {c A }} \\
64.71 \pm 0.07^{\text {c A }} \\
61.41 \pm 0.16^{\text {b A }} \\
60.01 \pm 0.28^{\text {a A }}\end{array}$ & $\begin{array}{c}7 \\
64.22 \pm 0.09^{\text {c A }} \\
64.77 \pm 0.23^{\text {c A }} \\
62.60 \pm 0.29^{\text {b B }} \\
60.61 \pm 0.15^{\text {a A }}\end{array}$ & $\begin{array}{c}14 \\
64.67 \pm 0.30^{\text {c A }} \\
65.22 \pm 0.19^{\text {c B }} \\
63.07 \pm 0.26^{\text {b B }} \\
61.30 \pm 0.24^{\text {a B }} \\
\text { Redness a* }^{*}\end{array}$ & $\begin{array}{c}21 \\
66.04 \pm 0.42^{\text {с B }} \\
65.46 \pm 0.21^{\text {с B }} \\
62.75 \pm 0.36^{\text {b B }} \\
60.30 \pm 0.45^{\text {a A }}\end{array}$ & $\begin{array}{c}28 \\
65.21 \pm 0.28^{\mathrm{d} \mathrm{A}, \mathrm{B}} \\
65.29 \pm 0.37^{\mathrm{d} \mathrm{B}} \\
62.94 \pm 0.15^{\mathrm{b} \mathrm{B}} \\
60.67 \pm 0.21^{\text {a A }}\end{array}$ \\
\hline $\begin{array}{l}\text { Day } \\
\text { CS } \\
\text { NS } \\
\text { CLS } \\
\text { CBS }\end{array}$ & $\begin{array}{c}0 \\
4.44 \pm 0.03^{\text {a C }} \\
8.91 \pm 0.07^{\text {с B,C }} \\
9.38 \pm 0.11^{\text {с A }} \\
11.65 \pm 0.09^{\text {d C }}\end{array}$ & $\begin{array}{c}7 \\
4.53 \pm 0.16^{\text {a C }} \\
8.47 \pm 0.45^{\text {c B }} \\
9.61 \pm 0.20^{\text {c A }} \\
10.41 \pm 0.28^{\text {d A }}\end{array}$ & $\begin{array}{c}14 \\
5.04 \pm 0.39 \text { a C } \\
9.48 \pm 0.22^{\mathrm{b} \mathrm{C}} \\
10.43 \pm 0.14^{\text {с B }} \\
11.44 \pm 0.03^{\text {с в }} \\
\text { Yellowness b* }\end{array}$ & $\begin{array}{c}21 \\
3.64 \pm 0.24^{\text {a B }} \\
9.58 \pm 0.10^{\mathrm{bC}} \\
10.39 \pm 0.17^{\mathrm{c} \mathrm{B}} \\
11.96 \pm 0.16^{\mathrm{dC}}\end{array}$ & $\begin{array}{c}28 \\
3.13 \pm 0.21^{\text {a A }} \\
7.86 \pm 0.18^{\text {b A }} \\
9.29 \pm 0.23^{\text {c A }} \\
10.31 \pm 0.03^{\text {d A }}\end{array}$ \\
\hline $\begin{array}{l}\text { Day } \\
\text { CS } \\
\text { NS } \\
\text { CLS } \\
\text { CBS }\end{array}$ & $\begin{array}{c}0 \\
15.36 \pm 0.29^{\text {c A }} \\
12.74 \pm 0.08^{\text {a A }} \\
14.69 \pm 0.35^{\text {b A }} \\
12.92 \pm 0.16^{\text {a A }}\end{array}$ & $\begin{array}{c}7 \\
15.77 \pm 0.11^{\text {с A }} \\
13.65 \pm 0.16^{\text {a B }} \\
15.88 \pm 0.27^{\text {с В }} \\
14.46 \pm 0.14^{\text {b B }}\end{array}$ & $\begin{array}{c}14 \\
15.74 \pm 0.18^{\mathrm{d} \mathrm{A}} \\
12.79 \pm 0.33^{\mathrm{a} \mathrm{A}} \\
15.03 \pm 0.19^{\mathrm{d} \mathrm{A}} \\
14.02 \pm 0.30^{\mathrm{c} \mathrm{A}, \mathrm{B}} \\
\Delta \mathrm{E}\end{array}$ & $\begin{array}{c}21 \\
15.77 \pm 0.03^{\text {b A }} \\
13.34 \pm 0.17^{\text {a B }} \\
15.27 \pm 0.37^{\text {b A }} \\
13.46 \pm 0.42^{\text {a A }}\end{array}$ & $\begin{array}{c}28 \\
16.23 \pm 0.08^{\mathrm{d} \mathrm{B}} \\
13.53 \pm 0.16^{\text {a B }} \\
15.38 \pm 0.22^{\text {c A,B }} \\
14.00 \pm 0.26^{\text {a A,B }}\end{array}$ \\
\hline $\begin{array}{l}\text { Day } \\
\text { CS } \\
\text { NS } \\
\text { CLS } \\
\text { CBS }\end{array}$ & $\begin{array}{l}0 \\
- \\
- \\
- \\
-\end{array}$ & $\begin{array}{c}7 \\
0.73 \pm 0.05^{\text {a A }} \\
1.01 \pm 0.11^{\text {a B }} \\
1.70 \pm 0.03^{\mathrm{bC}} \\
2.07 \pm 0.06^{\mathrm{cC}}\end{array}$ & $\begin{array}{c}14 \\
0.67 \pm 0.18^{\text {a A }} \\
1.40 \pm 0.04^{\mathrm{b} \mathrm{C}} \\
1.27 \pm 0.09^{\mathrm{b} \mathrm{B}} \\
1.31 \pm 0.20^{\mathrm{b} \mathrm{A}}\end{array}$ & $\begin{array}{c}21 \\
1.96 \pm 0.12^{\text {c C }} \\
0.61 \pm 0.15^{\text {a A }} \\
0.40 \pm 0.03^{\text {a A }} \\
1.26 \pm 0.08^{\text {b A }}\end{array}$ & $\begin{array}{c}28 \\
1.07 \pm 0.10^{\text {a B }} \\
1.74 \pm 0.04^{\text {b D }} \\
1.11 \pm 0.09^{\text {a B }} \\
1.78 \pm 0.03^{\text {b B }}\end{array}$ \\
\hline
\end{tabular}

CS, sausages with salt; NS, sausages with curing salt; CLS, sausages with Cistus incanus extract and lycopene; CBS, sausages with Cistus incanus extract and betanin; ${ }^{\mathrm{a}-\mathrm{d}}$, significant differences between the sausage variants (in columns) $(p<0.05)$; ${ }^{\mathrm{A}-\mathrm{D}}$, significant differences during storage (in rows) $(p<0.05)$.

Table 7. Effect of $C$. incanus extract with lycopene and betanin on the microbiological quality of pork sausages $(n=3)$.

\begin{tabular}{|c|c|c|c|c|c|c|c|}
\hline \multicolumn{8}{|c|}{$\mathrm{TMC}[\mathrm{cfu} / \mathrm{g}]$} \\
\hline day & 0 & 3 & 5 & 7 & 14 & 21 & 28 \\
\hline $\mathrm{CS}$ & $2.43 \pm 0.05^{\mathrm{bA}}$ & $3.85 \pm 0.12^{\mathrm{Bb}}$ & $4.64 \pm 0.13^{\mathrm{Cb}}$ & $6.72 \pm 0.06^{\mathrm{Da}}$ & $6.94 \pm 0.04^{\mathrm{Da}}$ & $7.91 \pm 0.14^{\mathrm{Eb}}$ & $7.70 \pm 0.10^{E}$ \\
\hline NS & $2.38 \pm 0.08^{\mathrm{bA}}$ & $2.48 \pm 0.03^{\mathrm{a} A}$ & $3.90 \pm 0.04^{\mathrm{a} \mathrm{B}}$ & $6.81 \pm 0.10^{a \mathrm{E}}$ & $6.72 \pm 0.07^{\mathrm{a} E}$ & $6.95 \pm 0.03^{a E}$ & $6.59 \pm 0.05^{a \mathrm{E}}$ \\
\hline CLS & $1.78 \pm 0.11^{\mathrm{a} \mathrm{A}}$ & $2.97 \pm 0.15^{a \mathrm{~B}}$ & $4.40 \pm 0.17^{\mathrm{bD}}$ & $6.15 \pm 0.13^{a \mathrm{~F}}$ & $6.80 \pm 0.10^{\text {a F }}$ & $7.53 \pm 0.05^{b G}$ & $7.34 \pm 0.07^{b G}$ \\
\hline CBS & $2.30 \pm 0.05^{\mathrm{bA}}$ & $3.30 \pm 0.07^{\mathrm{bB}}$ & $5.12 \pm 0.02^{\mathrm{cD}}$ & $6.93 \pm 0.11^{\mathrm{aE}}$ & $6.54 \pm 0.08^{\mathrm{aE}}$ & $7.40 \pm 0.02^{b F}$ & $7.56 \pm 0.17^{b F}$ \\
\hline \multicolumn{8}{|c|}{$\mathrm{LAB}[\mathrm{cfu} / \mathrm{g}]$} \\
\hline day & 0 & 3 & 5 & 7 & 14 & 21 & 28 \\
\hline $\mathrm{CS}$ & $2.28 \pm 0.09^{b A}$ & $3.01 \pm 0.05^{\mathrm{bB}}$ & $4.63 \pm 0.09^{b C}$ & $6.76 \pm 0.14^{\mathrm{a} E}$ & $7.21 \pm 0.12^{b F}$ & $8.15 \pm 0.06^{\mathrm{cG}}$ & $8.48 \pm 0.04^{\mathrm{cG}}$ \\
\hline NS & $2.51 \pm 0.03^{\mathrm{bA}}$ & $2.11 \pm 0.18^{a A}$ & $3.70 \pm 0.15^{\mathrm{a} \mathrm{B}}$ & $6.72 \pm 0.03^{a \mathrm{E}}$ & $6.77 \pm 0.07^{\mathrm{a} E}$ & $7.90 \pm 0.02^{b F}$ & $7.34 \pm 0.09 \mathrm{bF}$ \\
\hline CLS & $1.90 \pm 0.14^{\mathrm{a} \mathrm{A}}$ & $2.65 \pm 0.07^{\mathrm{a} \mathrm{B}}$ & $4.15 \pm 0.04^{\mathrm{bD}}$ & $6.23 \pm 0.10^{a \mathrm{~F}}$ & $7.12 \pm 0.15^{b G}$ & $6.57 \pm 0.13^{a \mathrm{~F}}$ & $7.02 \pm 0.11^{b G}$ \\
\hline CBS & $1.95 \pm 0.11^{\mathrm{a} \mathrm{A}}$ & $2.93 \pm 0.14^{\mathrm{bB}}$ & $5.19 \pm 0.08^{\mathrm{cD}}$ & $6.89 \pm 0.06^{\mathrm{a} E}$ & $7.19 \pm 0.08^{b F}$ & $6.67 \pm 0.05^{\mathrm{a} E}$ & $6.86 \pm 0.16^{\mathrm{a} E}$ \\
\hline \multicolumn{8}{|c|}{ TPC $[\mathrm{cfu} / \mathrm{g}]$} \\
\hline day & 0 & 3 & 5 & 7 & 14 & 21 & 28 \\
\hline $\mathrm{CS}$ & $2.26 \pm 0.16^{\mathrm{bA}}$ & $3.30 \pm 0.11^{\mathrm{bB}}$ & $3.85 \pm 0.07^{a \mathrm{~B}}$ & $6.91 \pm 0.14^{\mathrm{aE}}$ & $7.45 \pm 0.08^{b F}$ & $7.42 \pm 0.11^{b F}$ & $7.57 \pm 0.07^{b F}$ \\
\hline NS & $2.00 \pm 0.04^{\mathrm{bA}}$ & $2.42 \pm 0.02^{\mathrm{aA}}$ & $3.00 \pm 0.05^{\mathrm{a} B}$ & $6.83 \pm 0.18^{a D}$ & $6.93 \pm 0.06^{\mathrm{aD}}$ & $6.78 \pm 0.03^{\mathrm{a} D}$ & $6.28 \pm 0.17^{\mathrm{a} D}$ \\
\hline CLS & $1.48 \pm 0.09^{\mathrm{a} \mathrm{A}}$ & $2.40 \pm 0.16^{a \mathrm{~B}}$ & $3.30 \pm 0.12^{a C}$ & $6.26 \pm 0.03^{a \mathrm{E}}$ & $7.06 \pm 0.10^{\text {a F }}$ & $7.41 \pm 0.14^{\mathrm{bF}}$ & $7.21 \pm 0.02^{b F}$ \\
\hline CBS & $1.30 \pm 0.05^{\mathrm{a} \mathrm{A}}$ & $3.13 \pm 0.13^{b C}$ & $3.70 \pm 0.06^{\mathrm{aD}}$ & $6.90 \pm 0.09^{\mathrm{a} E}$ & $7.48 \pm 0.04^{\mathrm{bF}}$ & $7.37 \pm 0.12^{b F}$ & $7.60 \pm 0.10^{b F}$ \\
\hline \multicolumn{8}{|c|}{ Enterobacteriaceae $[\mathrm{cfu} / \mathrm{g}]$} \\
\hline day & 0 & 3 & 5 & 7 & 14 & 21 & 28 \\
\hline CS & $<1$ & $1.70 \pm 0.05^{\mathrm{a} A}$ & $2.32 \pm 0.16^{\mathrm{a} B}$ & $4.38 \pm 0.04^{b D}$ & $5.95 \pm 0.07^{\mathrm{cE}}$ & $5.58 \pm 0.03^{\mathrm{bE}}$ & $5.85 \pm 0.17^{\mathrm{bE}}$ \\
\hline NS & $<1$ & $1.30 \pm 0.09^{a A}$ & $2.04 \pm 0.02^{a b}$ & $4.20 \pm 0.11^{b D}$ & $4.23 \pm 0.14^{b D}$ & $4.28 \pm 0.05^{\mathrm{a} D}$ & $4.81 \pm 0.02^{a \mathrm{D}}$ \\
\hline CLS & $<1$ & $\begin{array}{c}2.00 \pm 0.14 \\
\mathrm{a}, \mathrm{b} \mathrm{A}\end{array}$ & $2.46 \pm 0.07$ a A & $3.48 \pm 0.15^{\text {a B }}$ & $3.00 \pm 0.03^{a B}$ & $4.72 \pm 0.08^{\mathrm{aC}}$ & $4.69 \pm 0.14^{\text {a C }}$ \\
\hline CBS & $<1$ & $2.79 \pm 0.06^{\mathrm{bA}}$ & $2.85 \pm 0.08^{a A}$ & $3.85 \pm 0.09^{a \mathrm{~B}}$ & $4.28 \pm 0.12^{b C}$ & $4.72 \pm 0.04^{\mathrm{aC}}$ & $5.16 \pm 0.10^{\mathrm{bD}}$ \\
\hline
\end{tabular}

CS, sausages with salt; NS, sausages with curing salt; CLS, sausages with Cistus incanus extract and lycopene; CBS, sausages with Cistus incanus extract and betanin; ${ }^{\mathrm{a}-\mathrm{c}}$, significantly differences between the sausage variants (in columns); ${ }^{\mathrm{A}-\mathrm{F}}$, significant differences during storage (in rows) $(p<0.05)$. The colors indicate the values from the highest (red) through medium (yellow) to the lowest (green). 


\subsection{Effect of Polyphenolic Extract and Dyes on Sensory Evaluation of Sausages}

At the time of production, the sausages with 1.8\% curing salt (NS), as well as the sausages with $C$. incanus extract and lycopene (CLS), were rated by the panel as being desirable in terms of taste (Table 8, Figure 2). A slightly lower, but equally high score was given to the sausage enriched with $C$. incanus extract and beetroot. The sausage with $1.8 \%$ salt was rated lowest. After 5 days of storage, the panel rated the sausage with C. incanus extract and lycopene (CLS) the highest in terms of taste (desirable).

In terms of odor, at the time of production the sausages with $1.8 \%$ curing salt (NS), as well as the sausage with C. incanus extract and lycopene (CLS), were characterized by an exceptionally desirable aroma. Other sausage variants were considered desirable. After 5 days of storage, the product with $2 \%$ curing salt (NS) content was again evaluated most favorably. The sausage with added lycopene was rated the highest among all tested variants in terms of odor after the second week of storage. Three weeks after the sausages were made, the odors of all the meat products were judged to be undesirable. After the last week of storage, the odor was rated extremely undesirable. Throughout the storage period, the products with added C. incanus extract and lycopene (CLS) and 1.8\% curing salt (NS) were characterized by the highest desirability in terms of color. The sausage with $1.8 \%$ salt (CS) was rated the lowest. At the time of manufacture, the color of the sausages with $1.8 \%$ curing salt content (NS) and C. incanus extract with lycopene (CLS) were rated extremely desirable, while products with $C$. incanus extract and beetroot (CBS) and $1.8 \%$ salt content (CS) were rated desirable. After four weeks of storage, the color of the sausage with $1.8 \%$ salt (CS) and the sausage enriched with $C$. incanus extract and beetroot (CBS) was considered extremely undesirable.

Table 8. Sensory analysis of the sausage variants during storage.

\begin{tabular}{|c|c|c|c|c|c|c|c|c|c|c|}
\hline $\begin{array}{c}\text { Storage Time } \\
\text { [Day] }\end{array}$ & 0 & 5 & 14 & 21 & 28 & 0 & 5 & 14 & 21 & 28 \\
\hline Sausage variant & \multicolumn{5}{|c|}{ Taste } & \multicolumn{5}{|c|}{ Odor } \\
\hline CS & 4.5 & 3.4 & n.t. & n.t. & n.t. & 4.8 & 3.6 & 3.0 & 2.4 & 1.0 \\
\hline NS & 5.0 & 3.5 & n.t. & n.t. & n.t. & 5.0 & 4.0 & 3.2 & 2.7 & 1.4 \\
\hline CLS & 5.0 & 4.0 & n.t. & n.t. & n.t. & 5.0 & 3.8 & 3.4 & 2.5 & 1.0 \\
\hline CBS & 4.8 & 3.6 & n.t. & n.t. & n.t. & 4.6 & 3.8 & 2.7 & 2.1 & 1.0 \\
\hline Sausage variant & \multicolumn{5}{|c|}{ Color } & \multicolumn{5}{|c|}{ Appearance } \\
\hline CS & 4.5 & 4.0 & 3.5 & 2.2 & 1.0 & 4.6 & 4.0 & 3.3 & 2.2 & 1.0 \\
\hline NS & 5.0 & 4.5 & 4.0 & 3.6 & 2.5 & 5.0 & 4.5 & 4.0 & 3.4 & 2.6 \\
\hline CLS & 5.0 & 4.5 & 4.2 & 3.5 & 2.4 & 5.0 & 4.5 & 4.0 & 3.3 & 2.5 \\
\hline CBS & 4.6 & 4.0 & 3.8 & 2.6 & 1.5 & 4.8 & 4.2 & 3.5 & 2.6 & 1.8 \\
\hline
\end{tabular}

CS, control sausages with salt; NS, sausages with curing salt; CLS, sausages with Cistus incanus extract and lycopene; CBS, sausages with Cistus incanus extract and beetroot; n.t., not tested.

On average, the sausages received similar scores in terms of the assessment of color. After the storage period, the sausage enriched with C. incanus extract and lycopene (CLS) and the sausage with $1.8 \%$ curing salt (NS) were rated the highest. The appearance of the sausage with the addition of $C$. incanus and beetroot (CBS) extract was rated slightly lower. The sausage with $1.8 \%$ salt (CS) content was the least desirable. Overall, in terms of flavor, odor, color, and appearance, the sausages with C. incanus extract and lycopene (CLS) and the sausages with $2 \%$ curing salt (NS) were rated the highest.

Swastike et al. [49] studied the effects of the addition of beetroot powder on the sensory characteristics of chicken sausages with tapioca flour as filler. Four ratios of beetroot powder and tapioca were used: 18:0, 17:1,16:2, and 15:3 [\%:\%]. Organoleptic analysis of the chicken sausages was performed by a panel of 25 people. The panel evaluated the taste, color, texture, and tenderness of the sausages. The use of $2 \%$ beetroot powder had the most beneficial effect on the color, taste, and texture of the meat products. Aykin-Dicer et al. [50] showed that enrichment with beetroot extract and beetroot powder can have a beneficial effect on the sensory attributes (color, odor and taste) of sausages, concluding that natural 
beetroot powder can be an alternative to carmine, a synthetic dye. Eyiler and Oztan [51] analyzed the effect of using tomato powder, a natural lycopene-rich colorant, on the taste, aroma, color, and overall desirability of sausage frankfurters. Organoleptic analysis of the products was conducted by a panel of 10 people. The addition of the natural colorant (lycopene) to the frankfurters had a positive impact on the organoleptic qualities of the product, which was characterized by high desirability in terms of taste, color, and odor.

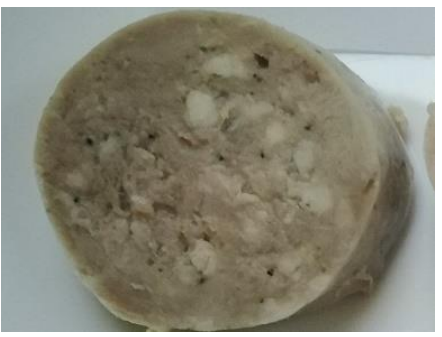

CS

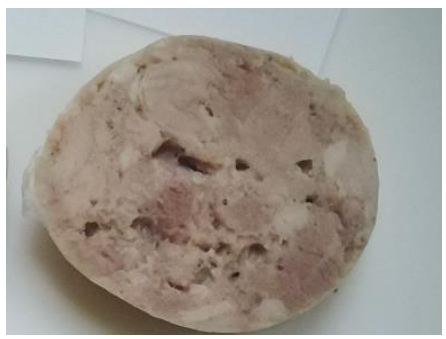

CLS

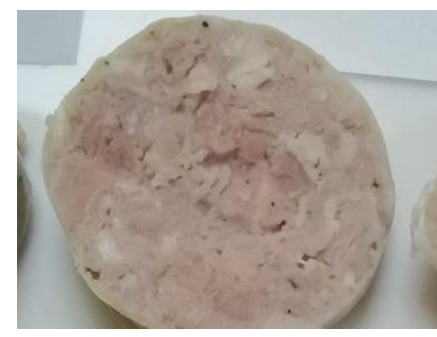

NS

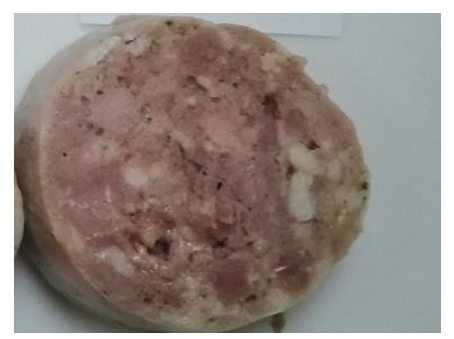

CBS

Figure 2. Sausages after 3 days of storage: CS, sausages with salt; NS, sausages with curing salt (containing nitrates); CLS, sausages with Cistus incanus extract and lycopene; CBS, sausages with Cistus incanus extract and betanin.

In our study, we confirmed high antioxidant activity of Cistus incanus extract resulting from the presence of many biologically active compounds. Many polyphenolic compounds were identified in which flavonoids were the largest group. When assessing the antimicrobial effect of Cistus incanus extract on selected bacteria, it was shown that increasing the concentration of the extracts decreased the growth rate of E. coli, P. fragi, S. enterica, $B$. thermosphacta, L. sakei, L. monocytogenes to an increasing extent. C. incanus extract also prolonged the duration of lag phase. The dyes lycopene and betanin affected the growth parameters of the bacteria tested. No synergistic effect of the extract with dyes was noted. The use of $C$. incanus extract and lycopene and betanin dyes effectively slowed down the lipid oxidation process in sausages. This is due to the presence of polyphenols in the plant extract. Therefore, Cistus extract in combination with dyes delays unfavorable changes in the product and allow to maintain the desired aroma and taste. Cistus incanus extract, lycopene and betanin significantly increase the proportion of red color, which is important information for consumers because the content of red color in meat products is a feature of their quality and freshness. From the microbiological point of view, the use of $C$. incanus extract and dyes and a $50 \%$ reduction in nitrate content did not deteriorate the quality of the sausages. In the sensory evaluation, the consumers positively assessed the sausages with the addition of Cistus incanus extract and natural dyes. However, they rated the sausage with $C$. incanus extract and lycopene the best.

\section{Conclusions}

In response to consumer demand for natural ingredients and the elimination of chemical additives, natural plant-based agents are being sought to replace commonly-used preservatives. Plants and especially plant extracts offer a promising alternative and exhibit a variety of properties including antimicrobial activity. In this study, C. incanus extract 
in combination with the natural pigments lycopene (from tomatoes) and betanin (from beetroot) was found to exhibit antioxidant properties in vacuum-packed pork sausages, as well as giving a red color to the meat products. However, the additives did not show antimicrobial properties in the tested amounts. Further optimization of the dose is needed to achieve an antimicrobial effect.

Author Contributions: Conceptualization, M.S.; methodology, M.S., A.N., A.C. (Agata Czyżowska), I.G.-C., and and A.C. (Aleksandra Czerbniak); formal analysis, M.S., A.N., A.C. (Agata Czyżowska), and I.G.-C.; data curation, M.S., A.N.; writing—original draft preparation, M.S., A.N., A.C. (Agata Czyżowska), I.G.-C., and A.C. (Aleksandra Czerbniak). All authors have read and agreed to the published version of the manuscript.

Funding: This research received no external funding.

Institutional Review Board Statement: Not applicable.

Informed Consent Statement: Not applicable.

Data Availability Statement: Data is contained within the article.

Conflicts of Interest: The authors declare no conflict of interest.

\section{References}

1. Sucu, C.; Turp, G.Y. The investigation of the use of beetroot powder in Turkish fermented beef sausage (sucuk) as nitrite alternative. Meat Sci. 2018, 140, 158-166. [CrossRef]

2. Honikel, K.O. The use and control of nitrate and nitrite for the processing of meat products. Meat Sci. 2008, 78, 68-76. [CrossRef] [PubMed]

3. Zhang, H.; Sun, C.; Han, W.; Zhang, J.; Hou, J. Analysis of the monitoring status of residual nitrite in meat products in China from 2000 to 2011. Meat Sci. 2018, 136, 30-34. [CrossRef]

4. Jin, S.K.; Choi, J.S.; Yang, H.S.; Park, T.S.; Yim, D.G. Natural curing agents as nitrite alternatives and their effects on the physicochemical, microbiological properties and sensory evaluation of sausages during storage. Meat Sci. 2018, 146, 34-40. [CrossRef] [PubMed]

5. Lorenzo, J.M.; Pateiro, M.; Domínguez, R.; Barba, F.J.; Putnik, P.; Kovačević, D.B.; Franco, D. Berries extracts as natural antioxidants in meat products: A review. Int. Food Res. J. 2018, 106, 1095-1104. [CrossRef] [PubMed]

6. Dominguez, R.; Pateiro, M.; Agregán, R.; Lorenzo, J.M. Effect of the partial replacement of pork back fat by microencapsulated fish oil or mixed fish and olive oil on the quality of frankfurter type sausage. J. Food Sci. Technol. 2017, 54, 26-37. [CrossRef] [PubMed]

7. Coutinho de Oliveira, T.L.; Malfitano de Carvalho, S.; de Araujo Soares, R.; Apericida Andrade, M.; das Graças Cardoso, M.; Mendes Ramos, E.; Hilsdorf Piccolia, R. Antioxidant effects of Satureja montana L. essential oil on TBARS and color of mortadellatype sausages formulated with different levels of sodium nitrite. LWT Food Sci. Technol. 2012, 45, 204-212. [CrossRef]

8. S Šojić, B.; Pavlić, B.; Ikonić, P.; Tomović, V.; Ikonić, B.; Zeković, Z.; Kocić-Tanackova, S.; Jokanovića, M.; Škaljaca, S.; Ivića, M. Coriandere ssential oil as natural food additive improves quality and safety of cooked pork sausages with different nitrite levels. Meat Sci. 2019, 10, 78-79. [CrossRef]

9. Lin, Y.; Huang, M.; Zhou, G.; Zou, Y.; Xu, X. Prooxidant effects of the combination of green tea extract and sodium nitrite for accelerating lipolysis and lipid oxidation in pepperoni during storage. J. Food Sci. 2011, 76, C694-C700. [CrossRef]

10. Riazi, F.; Zeynail, F.; Hoseini, E.; Behmadi, H.; Savadkoohi, S. Oxidation phenomena and color properties of grape pomace on nitrite-reduced meat emulsion systems. Meat Sci. 2016, 121, 350-358. [CrossRef]

11. Semeriak, K.; Jarmoluk, A. Wpływ naturalnych antyoksydantów na barwę peklowanych przetworów mięsnych. Żywn. Nauka. Technol. Jakość 2011, 4, 138-150.

12. Hammad, H.M.H.; Ma, M.; Jin, G.; Khalifa, I.; Zeng, Q.; Liu, Y. Nitroso-Hemoglobin increased the color stability and inhibited the pathogenic bacteria in a minced beef model: A combined low-field NMR study. Food Sci. Anim. Resour. 2019, 39, 704-724. [CrossRef]

13. Sikorski, Z. Chemia Żywności-Składniki Żywności Tom 1; Wydawnictwo Naukowo-Techniczne: Warszawa, Poland, 2007; pp. $142-170$.

14. Sigurdson, G.T.; Tang, P.; Giusti, M.M. Natural Colorants: Food Colorants from Natural Sources. Annu. Rev. Food Sci. Technol. 2017, 8, 261-280. [CrossRef]

15. Czajkowska, K.; Kowalska, H.; Piotrowski, D. Rola konsumenta w procesie projektowania nowych produktów spożywczych. Zesz. Probl. Post. Nauk Roln. 2013, 575, 23-32.

16. Tabaka, K.; Cierach, M. Barwa modelowych produktów mięsnych z dodatkiem preparatów zawierających barwniki naturalne i o zmniejszonej zawartości azotanu III sodowego. Zesz. Probl. Post. Nauk. Roln. 2014, 576, 161-171. 
17. Dias, S.; Castanheira, E.M.S.; Gil Fortes, A.; Pereira, D.M.; Rodrigues, R.O.; Pereira, R.; Gonçalves, M.S.T. Application of Natural Pigments in Ordinary Cooked Ham. Molecules 2020, 25, 2241. [CrossRef]

18. Østerlie, M.; Lerfall, J. Lycopene from tomato products added minced meat: Effect on storage quality and colour. Int. Food Res. J. 2005, 38, 925-929. [CrossRef]

19. Wittpahl, G.; Kölling-Speer, I.; Basche, S.; Herrmann, E.; Hannig, M.; Speer, K.; Hannig, K. The polyphenolic composition of Cistus incanus herbal tea and its antibacterial and antiadherent activity against Streptococus mutans. Planta Med. 2015, 81, 1727. [CrossRef]

20. Rebensburg, S.; Helfer, M.; Schneider, M.; Koppensteiner, H.; Eberle, J.; Schindler, M.; Gürtler, L.; Brack-Werner, R. Potent in vitro antiviral acitivity of Cistus incanus extract against HIV and Filoviruses targets viral envelope proteins. Sci. Rep. Nat. 2016, 6, 20394. [CrossRef]

21. Stępień, A.; Aebisher, D.; Bartusik-Aebisher, D. Biological properties of Cistus species. Eur. J. Clin. Exp. Med. 2018, 16, 127-132. [CrossRef]

22. Vitali, F.; Pennisi, G.; Attaguile, G.; Savoca, F. Antiproliferative and cytotoxic activity of extracts from Cistus incanus L. and Cistus monspeliensis L. on human prostate cel lines. Nat. Prod. Res. 2011, 25, 188-202. [CrossRef]

23. Kozłowska, M. Badanie zawartości polifenoli i aktywności przeciwutleniającej ekstraktów z roślin przyprawowych podczas ich przechowywania. Bromatol. Chem. Toksykol. 2012, 3, 358-360.

24. Tarladgis, B.G.; Watts, B.M.; Younathan, M.T.; Dugan, L., Jr. A distillation method for the quantitative determination of ma-lonaldehyde in rancid foods. J. Am. Oil Chem. Soc. 1960, 37, 44-48. [CrossRef]

25. ISO 6887-2:2017 Microbiology of the Food Chain-Preparation of Test Samples, Initial Suspension and Decimal Dilutions for Microbiological Examination-Part 2: Specific Rules for the Preparation of Meat and Meat Products; ISO: Geneva, Switzerland, 2017.

26. Floegel, A.; Dae-Ok, K.; Sang-Jin, C.; Koo, S.I.; Chun, O.K. Comparison of ABTS /DPPH assays to measure antioxidant capacity in popular antioxidant-rich US foods. J. Food Compos. Anal. 2011, 24, 1043-1048. [CrossRef]

27. Sayah, K.; Marmouzi, I.; Naceiri Mrabti, H.; Cherrah, Y. Antioxidant Activity and Inhibitory Potential of Cistus saliifolius (L) and Cistus monospeliensis (L). Aerial Parts Extracts against Key Enzymes Linked to Hyperglycemia. J. Biomed. Biotechnol. 2017, 2017, 1-7. [CrossRef]

28. Koubaier, H.; Snoussi, A.; Essaidi, I.; Chaabouni, M.M.; Thonart, P.; Bouzouita, N. Betalain and Phenolic Compositions, Antioxidant Activity of Tunisian Red Beet (Beta vulgaris L. conditiva) Roots and Stems Extracts. J. Food Prop. 2014, 17, 1934-1945. [CrossRef]

29. Jaswir, I.; Noviendri, D.; Hasrini, R.F.; Octavianti, F. Carotenoids: Sources, medicinal properties and their application in food and nutraceutical industry. J. Med. Plant Res. 2011, 5, 7119-7131. [CrossRef]

30. Zuorro, A.; Lavecchia, R.; Medici, F.; Piga, L. Enzyme-assisted production of tomato seed oil enriched with lycopene from tomato pomace. Food Bioprocess Technol. 2012, 6, 3499-3509. [CrossRef]

31. Caseiro, M.; Ascenso, A.; Costa, A.; Creagh-Flynn, J.; Johnson, M.; Simões, S. Lycopene in human health. LWT Food Sci. Technol. 2020, 127, 109323. [CrossRef]

32. Barrajón-Catalán, E.; Fernández-Arroyo, S.; Roldán, C.; Guillén, E.; Saura, D.; Segura-Carretero, A.; Micol, V. A systematic study of the polyphenolic composition of aqueous extracts deriving from several Cistus genus species: Evolutionary relationship. Phytochem. Anal. 2011, 22, 303-312. [CrossRef]

33. Riehle, P.; Vollmer, M.; Rohn, S. Phenolic compounds in Cistus incanus herbal infusions-Antioxidant capacity and thermal stability during the brewing proces. Int. Food Res. J. 2013, 53, 891-899. [CrossRef]

34. Bouamama, H.; Noel, T.; Villard, J.; Benharref, A.; Jana, M. Antimicrobial acti-vites of the leafextracts of two Moroccan cistus L. species. J. Ethnopharmacol. 2006, 104, 104-107. [CrossRef]

35. Rajnbart Nedamani, A.; Rajnbart Nedemani, E. Brief report Antimicrobial Property of Lycopene Oleoresin on some Food Pathogens Running Head: Lycopene oleoresin antibacterial potent. Iran. J. Sci. Technol. Trans. A Sci. 2016, 12, $382-387$.

36. Efenberger-Szmechtyk, M.; Gałązka-Czarnecka, I.; Otlewska, A.; Czyżowska, A.; Nowak, A. Aronia melanocarpa (Michx.) Elliot, Chaenomeles superba Lindl. and Cornus mas L. leaf extracts as natural preservatives for pork meat products. Molecules 2021, 26, 3009. [CrossRef]

37. Farhadi, F.; Khameneh, B.; Iranshahi, M.; Iranshahy, M. Antibacterial activity of flavonoids and their structure-activity relationship: An update review. Phytother. Res. 2019, 33, 13-40. [CrossRef] [PubMed]

38. Yoda, Y.; Hu, Z.-H.; Zhao, W.-H.; Shimamura, T. Different susceptibilities of Staphylococcus and gram-negative rods to epigallocatechin gallate. J. Infect. Chemother. 2004, 10, 55-58. [CrossRef] [PubMed]

39. Xu, Y.; Shi, C.; Wu, Q.; Zheng, Z.; Liu, P.; Li, G.; Peng, X.; Xia, X. Antimicrobial Activity of Punicalagin Against Staphylococcus aureus and Its Effect on Biofilm Formation. Foodborne Pathog. Dis. 2017, 14, 282-287. [CrossRef]

40. Kuchta, A.; Konopacka, A.; Waleron, K.; Viapiana, A.; Wesołowski, M.; Dąbkowski, K.; Ćwiklińska, A.; Mickiewicz, A.; Śledzińska, A.; Wieczorek, E.; et al. The effect of Cistus incanus herbal tea supplementation on oxidative stress markers and lipid profile in healthy adults. Cardiol. J. 2021, 28, 534-542. [CrossRef]

41. Viapiana, A.; Konopacka, A.; Waleron, K.; Wesolowski, M. Cistus incanus L. commercial products as a good source of polyphenols in human diet. Ind. Crop. Prod. 2017, 107, 297-304. [CrossRef]

42. Shan, B.; Cai, Y.Z.; Brooks, J.D.; Corke, H. The in vitro antibacterial activity of dietary spice and medicinal herb extracts. Int. J. Food Microbiol. 2007, 117, 112-119. [CrossRef] 
43. Taguri, T.; Tanaka, T.; Kouno, I. Antimicrobial activity of 10 different plant polyphenols against bacteria causing food-borne disease. Biol. Pharm. Bull. 2004, 27, 1965-1969. [CrossRef] [PubMed]

44. Plumed-Ferrer, C.; Väkeväinen, K.; Komulainen, H.; Rautiainen, M.; Smeds, A.; Raitanen, J.E.; Eklund, P.; Willför, S.; Alakomi, H.-L.; Saarela, M.; et al. The antimicrobial effects of wood-associated polyphenols on food pathogens and spoilage organisms. Int. J. Food Microbiol. 2013, 164, 99-107. [CrossRef]

45. Zhang, H.; Peng, X.; Li, X.; Wu, J. The application of clove extract protects Chinese-style sausages against oxidation and quality deterioration. Korean J. Food Sci. Anim. 2017, 31, 114-122. [CrossRef] [PubMed]

46. Deda, M.S.; Bloukas, J.G.; Fista, G.A. Effect of tomato paste and nitrite level on processing and quality characteristics of frankfurters. Meat Sci. 2007, 76, 501-508. [CrossRef]

47. Sośnicka, M.; Nowak, A.; Gałązka-Czarnecka, I. Wpływ ekstraktów z wierzbownicy drobnokwiatowej Epilobium parviflorum i czystka Cistus incanus na wybrane parametry produktów mięsnych. In Przegląd Badań z Zakresu Żywienia Technologii Żywności; Maciag, K., Maciag, M., Eds.; Tygiel: Lublin, Poland, 2019; pp. 20-31, ISBN 978-83-65932-88-4.

48. Nowak, A.; Czyżowska, A.; Efenberger- Szmechtyk, M.; Krala, L. Polyphenolic extracts of cherry (Prunus cerasus L.) and blackcurrant (Ribes nigrum L.) leaves as natural preservatives in meat products. Food Microbiol. 2016, 59, 142-149. [CrossRef]

49. Swastike, W.; Suryanto, E.; Rusman, R.; Hanim, C.; Jamhari, J.; Erwanto, Y.; Jumari, J. The Substitution Effects of Tapioca Starch and Beetroot Powder as Filler on The Physical and Sensory Characteristics Of Chicken Sausage. J. Ilmu dan Teknologi Hasil Ternak 2020, 15, 97-107. [CrossRef]

50. Aykin-Dinçer, E.; Güngör, K.K.; Çağlar, E.; Erbaş, M. The use of beetroot extract and extract powder in sausages as natural food colorant. Int. J. Food. Eng. 2021, 17, 75-82. [CrossRef]

51. Eyiler, E.; Oztan, A. Swastike Production of frankfurters with tomato powder as a natural additive. LWT Food Sci. Technol. 2011, 44, 304-307. [CrossRef] 